\title{
ON A THEOREM CONCERNING BAIRE FUNCTIONS
}

\author{
C. T. TUCKER
}

\begin{abstract}
Mazurkiewicz, Sierpiński, and Kempisty proved that a function in Baire class 1 is the uniform limit of a sequence of functions each of which is the difference of two upper semicontinuous functions. A generalization of this theorem is shown to be a consequence of order and linear properties alone.
\end{abstract}

Mazurkiewicz [2], Sierpiński [3], and Kempisty [1] proved that a function in Baire class 1 is the uniform limit of a sequence of functions each of which is the difference of two upper semicontinuous functions. It is shown here that this theorem is a consequence of order and linear properties alone.

Suppose $L$ is a Riesz space (lattice ordered vector space) which is $\sigma$ complete (every countable set of positive elements has a greatest lower bound). A sequence $f_{1}, f_{2}, \cdots$ of points is said to order converge to the point $f$ if there exists a sequence $u_{1} \geqq u_{2} \geqq u_{3} \geqq \cdots$ and a sequence $l_{1} \leqq l_{2} \leqq$ $l_{3} \leqq \cdots$ of points such that $\bigvee l_{p}=f, \wedge u_{p}=f$, and $l_{p} \leqq f_{p} \leqq u_{p}$. If $L$ is the space of all real valued functions on a set then order convergence is equivalent to pointwise convergence. If $L$ is an $L_{p}$ space or the set of all measurable functions on a measure space then order convergence is equivalent to boundedness and convergence almost everywhere.

A sequence $f_{1}, f_{2}, f_{3}, \cdots$ of points of $L$ is said to converge relatively uniformly to $f$ if there exists an element $g$ of $L$ (called the regulator) such that if $\varepsilon>0$, there exists a number $N_{\varepsilon}$ such that if $n$ is a positive integer greater than $N_{\varepsilon}$, then $-\varepsilon g \leqq f-f_{n} \leqq \varepsilon g$. Relative uniform convergence implies order convergence. Generally it is stronger.

Suppose $C$ is a Riesz subspace of $L$ and $U$ is the set of all elements which are the greatest lower bound of a countable subset of $C$. The Riesz space version of the theorem of Mazurkiewicz, Sierpiński, and Kempisty is that if $f$ is the order limit of a sequence of points of $C$ then it is the relative uniform limit of a sequence of points each of which is the difference of two points of $U$. This is not true in general, e.g. let $L$ be the space of all bounded functions on $[0,1]$ and $C$ be the space of all continuous functions

Received by the editors December 17, 1971 and, in revised form, November 20, 1972.

AMS (MOS) subject classifications (1970). Primary 26A21, 46A40.

Key words and phrases. Baire functions, Riesz space, order convergence, relative uniform convergence.

(c) American Mathematical Society 1973 
on $[0,1]$. In Example 1 near the end of this paper it is shown that there is a bounded function in Baire class 1 which is not the uniform limit of any sequence of functions each of which is the difference of two bounded upper semicontinuous functions. Thus additional assumptions are required, some of which are discussed here.

For an important class of Riesz spaces, the regular spaces, relative uniform convergence is equivalent to order convergence and the theorem of Mazurkiewicz, Sierpinski, and Kempisty is trivially true. For a definition of regular Riesz spaces and a discussion of some of their properties see [4]. It is sufficient to note here that the class of regular Riesz spaces includes the spaces $l_{p}(1 \leqq p<\infty)$ and the spaces $L_{p}(1 \leqq p<\infty)$ of $p$ th power summable functions with respect to a countably additive measure.

However if $L$ is the space of all real valued functions on an infinite set $X$, then $L$ is not regular, order convergence is weaker than relative uniform convergence, and the theorem of Mazurkiewicz, Sierpiński, and Kempisty is, in this instance, not immediate. This case will be covered in the theorem which follows.

Denote by $\theta$ the zero element of $L$. The point $e \geqq \theta$ of $L$ is said to be a weak unit if for each point $f$ of $L, f \wedge e=\theta$ only if $f=\theta$. The point $e \geqq \theta$ of $L$ is said to have property c if whenever $h_{1} \leqq h_{2} \leqq h_{3} \leqq \cdots$ is a sequence of elements of $L$ such that $e=\bigvee h_{i}$ then there exists a point $b$ of $L$ such that $b \leqq \sum_{i=1}^{n} h_{i}$ for every positive integer $n$.

It is shown in this paper that if $e$ has property $\mathrm{c}$ then it is a weak unit. It can be shown by use of a representation theorem that if $e$ is a weak unit of $L$ then $L$ can be embedded in a $\sigma$-complete Riesz space $V$ such that $e$ has property c in $V$.

THEOREM 1. Suppose $L$ is a $\sigma$-complete Riesz space, $e$ is a point of $L$ with property $\mathrm{c}, C$ is a Riesz subspace of $L$ containing $e$, and $U$ is the set of all points which are the greatest lower bounds of a countable subset of $C$. Then if $f$ is the order limit of a sequence of points of $C$, it is the relative uniform limit, with regulator $e$, of a sequence of points each of which is the difference of two points of $U$.

Proof. It will be sufficient to show that if $k$ is a positive number there is a point of $U-U$ in the order interval $[f, f+k e]$.

Let $f+k e=g$. There is a sequence $\left\{f_{p}\right\}$ of points of $C$ order converging to $f$. Let $y_{i}=\bigvee_{p \geqq i} f_{n}$ and $z_{i}=\bigwedge_{p \geqq i} f_{p}+k e$. Then $-y_{i} \in U$ and $z_{i} \in U$. Also $f=\Lambda y_{i}$ and $g=\bigvee z_{i}$.

Let $\alpha_{1}=-y_{1}$ and for each positive integer $i$ let

$$
\beta_{i}=\left(z_{i}-y_{i}\right) \wedge \theta \text { and } \alpha_{i+1}=\left(z_{i}-y_{i+1}\right) \wedge \theta .
$$

Each of $\alpha_{i}$ and $\beta_{i}$ is in $U$. Now $k e=\bigvee\left(z_{i}-y_{i}\right)$ and $k e=\bigvee\left(z_{i}-y_{i+1}\right)$. As $e$ 
has property c, $\left\{\sum_{j=1}^{n} \alpha_{j}\right\}$ and $\left\{\sum_{j=1}^{n} \beta_{j}\right\}$ each have a lower bound in $L$. By hypothesis there exists an $a \leqq \theta$ and a $b \leqq \theta$ both in $U$ such that

$$
b=\bigwedge \sum_{j=1}^{n} \beta_{j} \quad \text { and } \quad a=\bigwedge \sum_{j=1}^{n} \alpha_{j} .
$$

Now to show $g \geqq b-a \geqq f$.

Let $m_{i}=\sum_{j=1}^{i+1}-\alpha_{j}$ and $n_{i}=\sum_{j=1}^{i} \beta_{j}$.

To show that $b-a \geqq f$, the following facts will be derived:

(a) $n_{i+1}+m_{i+1} \leqq n_{i}+m_{i}$,

(b) $\wedge\left(n_{i}+m_{i}\right)=b-a$, and

(c) $n_{i}+m_{i} \geqq y_{i+1}$.

As $f=\bigwedge y_{i}$, statements (b) and (c) are all that is necessary. Statement (a) is used in the argument for statement (b).

The following identity, which is valid in all vector lattices, will be used repeatedly: $u-(v \vee u)=(u-v) \wedge \theta$.

(a) If $i$ is a positive integer

$$
\begin{aligned}
n_{i+1}+m_{i+1}-\left(n_{i}+m_{i}\right) & =\left(z_{i+1}-y_{i+1}\right) \wedge \theta-\left(z_{i+1}-y_{i+2}\right) \wedge \theta \\
& =z_{i+1}-\left(z_{i+1} \vee y_{i+1}\right)-z_{i+1}+\left(z_{i+1} \vee y_{i+2}\right) \\
& =\left(z_{i+1} \vee y_{i+2}\right)-\left(z_{i+1} \vee y_{i+1}\right) .
\end{aligned}
$$

Now $y_{i+2} \leqq y_{i+1}$, so $z_{i+1} \vee y_{i+2} \leqq z_{i+1} \vee y_{i+1}$. Thus $\theta \geqq n_{i+1}+m_{i+1}-\left(n_{i}+m_{i}\right)$ and $n_{i}+m_{i} \geqq n_{i+1}+m_{i+1}$.

(b) If $i$ is a positive integer,

$$
b+m_{i} \leqq b+m_{i+1} \leqq n_{i+1}+m_{i+1} \leqq n_{i}+m_{i} .
$$

Thus $n_{i}+m_{i}$ is an upper bound for $b+m_{p}$ and $n_{i}+m_{i} \geqq \bigvee\left(b+m_{p}\right)=$ $b-a$. Then as $b-a \leqq n_{i}+m_{i} \leqq n_{i}-a$ and $b-a=\wedge n_{i}-a$, it follows that $\bigwedge\left(n_{i}+m_{i}\right)=b-a$.

(c) If $i$ is a positive integer,

$$
\begin{aligned}
n_{i}+m_{i} & =y_{1}+\sum_{j=1}^{i}\left\{\left[\left(z_{j}-y_{j}\right) \wedge \theta\right]-\left[\left(z_{j}-y_{j+1}\right) \wedge \theta\right]\right\} \\
& =y_{1}+\sum_{j=1}^{i}\left\{\left[-y_{j}-\left(-y_{j} \vee-z_{j}\right)\right]-\left[-y_{j+1}-\left(-y_{j+1} \vee-z_{j}\right)\right]\right\} \\
& =y_{1}+\sum_{j=1}^{i}\left(y_{j+1}-y_{j}\right)+\sum_{j=1}^{i}\left[\left(-y_{j+1} \vee-z_{j}\right)-\left(-y_{j} \vee-z_{j}\right)\right] \\
& =y_{i+1}+\sum_{j=1}^{i}\left[\left(y_{j} \wedge z_{j}\right)-\left(y_{j+1} \wedge z_{j}\right)\right] .
\end{aligned}
$$


As $y_{j} \geqq y_{j+1}, y_{j} \wedge z_{j} \geqq y_{j+1} \wedge z_{j}$, and $\left[\left(y_{j} \wedge z_{j}\right)-\left(y_{j+1} \wedge z_{j}\right)\right] \geqq \theta$. So

$$
n_{i}+m_{i}=y_{i+1}+\sum_{j=1}^{i}\left[\left(y_{j} \wedge z_{j}\right)-\left(y_{j+1} \wedge z_{j}\right)\right] \geqq y_{i+1}+\sum_{j=1}^{i} \theta=y_{i+1} \text {. }
$$

This completes the proof that $b-a \geqq f$. An analogous argument shows that $b-a \leqq g$.

In the following two theorems the Riesz space $L$ is not necessarily assumed to be $\sigma$-complete but is Archimedean. The statement that $L$ is Archimedean means that for each point $f \geqq \theta$ of $L, \wedge(1 / n) f=\theta$. The property of $\sigma$-completeness implies the property of being Archimedean.

The proof for Theorem 2 was suggested by the referee. It is simpler then the author's original argument.

TheOrem 2. Suppose L is an Archimedean Riesz space and e is a point of $L$ with property $\mathrm{c}$. Then $e$ is a weak unit.

Proof. Suppose $x \in L$ and $x \wedge e=\theta$. Define $x_{n}=e-(1 / n) x$ for each positive integer $n$. Then $x_{1} \leqq x_{2} \leqq x_{3} \cdots$ and $\bigvee x_{n}=e$. Now for each positive integer $k, \sum_{n=1}^{k} x_{n}=k e-\sum_{n=1}^{k}(1 / n) x$. As $e$ has property c, there exists a point $b$ of $L$ such that $b \leqq k e-\sum_{n=1}^{k}(1 / n) x$. Since $x \wedge e=\theta, b \leqq k e$ and $b \leqq-\sum_{n=1}^{k}(1 / n) x$. Thus, $-b \geqq\left(\sum_{n=1}^{k} 1 / n\right) x$. The series $\sum 1 / n$ increases without bound, so that for every positive integer $p$ it is true that $-b \geqq p x$. Since $L$ is Archimedean, this implies that $x=\theta$.

The statement that $e$ is a strong unit of $L$ means that $e \geqq \theta$ and if $f$ is in $L$ there is a positive number $k$ such that $k e \geqq f$. If $e$ is a strong unit, then it is a weak unit.

In the following, by use of a representation theorem for Archimedean vector lattices, $L$ will be assumed to be a subspace of the set of all continuous extended real valued functions on an extremally disconnected compact Hausdorff space $S$ which are finite except possibly on a nowhere dense subset of $S$.

If $L$ is assumed to have a weak unit $e$, then $e$ can be taken to be the function identically equal to 1 on $S$. Further, if $L$ is assumed to have a strong unit then $L$ can be assumed to consist of only finite valued functions on $S$.

THeORem 3. Suppose $L$ is an Archimedean Riesz space, $e$ is a point of $L$ with property $\mathrm{c}$ and $g$ is a strong unit of $L$. Then $e$ is a strong unit and $g$ has property c. Further, if $L$ is $\sigma$-complete, it is finite dimensional.

Proof. As $g \geqq \theta,\{e-(1 / p) g\}$ is a nondecreasing sequence such that $\bigvee(e-(1 / p) g)=e$. Thus there is an element $k \in L$ such that $k \leqq \sum_{p=1}^{n} e-$ $(1 / p) g$ for every positive integer $n$. There is a negative integer $r$ such that 
$r g \leqq k$. So $\sum_{p=1}^{n} e-(1 / p) g=n e-g \sum_{p=1}^{n} 1 / p \geqq r g$. Or, $n e \geqq g\left(r+\sum_{p=1}^{n}(1 / p)\right)$. Now as mentioned above $g$ may be taken to be the function which is 1 at every point of $S$. Also, let $n$ be an integer such that $\sum_{p=1}^{n}(1 / p) \geqq-r+1$. Thus $n e \geqq\left(r+\sum_{p=1}^{n}(1 / p)\right) \geqq 1=g$. Therefore $e$ is a strong unit.

Let $g_{1} \leqq g_{2} \leqq g_{3} \leqq \cdots$ be a sequence of elements of $L$ such that $g=\vee g_{i}$. There exists a positive integer $k$ such that $k g \geqq e$. Then $\left\{\left[k\left(g_{i} \vee \theta\right)\right] \wedge\right.$ $\left.e+g_{i} \wedge \theta\right\}$ is a nondecreasing sequence with supremum $e$. Thus there is an element $a \in L$ such that $\sum_{i=1}^{\infty} g_{i} \wedge \theta \geqq a$.

Suppose $L$ is $\sigma$-complete and $e$ has property c, is a strong unit, and is equal to 1 at each point of $S$.

Let $\Omega$ be the collection of all subsets $\omega$ of $S$ such that the characteristic function of $\omega$ is in $L$. Each set in $\Omega$ is open and closed and if $\omega$ is in $\Omega$ then $\omega^{\prime}$ is in $\Omega$. Further the union and intersection of any two sets in $\Omega$ is in $\Omega$. Suppose $\omega_{1}, \omega_{2}, \omega_{3}, \cdots$ is a countable increasing tower of sets in $\Omega$. The characteristic function of $\operatorname{cl}\left(\bigcup \omega_{i}\right)$ is the supremum of the characteristic functions of $\omega_{1}, \omega_{2}, \cdots$ and must belong to $L$ as $L$ is $\sigma$ complete. Thus $\operatorname{cl}\left(\bigcup \omega_{i}\right)$ is in $\Omega$ as is $\omega_{0}=\left(\operatorname{cl}\left(\bigcup \omega_{i}\right)\right)^{\prime}$. Then $\omega_{1} \cup \omega_{0}, \omega_{2} \cup \omega_{0}$, $\omega_{3} \cup \omega_{0}, \cdots$ is an increasing tower of sets of $\Omega$ whose union is dense in $S$.

Suppose $x_{0}$ is a point of $S$ which is not in $\bigcup\left(\omega_{i} \cup \omega_{0}\right)$. For each positive integer $i$ let $f_{i}$ be the function which is 1 at each point of $\omega_{i} \cup \omega_{0}$ and -1 otherwise. Then $\bigvee f_{i}=e$ and each $f_{i}$ is in $L$. But as $\sum_{i=1}^{n} f_{i}\left(x_{0}\right) \rightarrow-\infty$ as $n \rightarrow \infty$ and each function in $L$ is bounded, there is no $a$ in $L$ such that $a \leqq \sum_{i=1}^{n} f_{i}$ for each positive integer $n$. This contradicts the fact that $e$ has property c. Therefore, $\cup\left(\omega_{i} \cup \omega_{0}\right)=S$. As $S$ is compact, some finite subtower covers $S$. This implies that the original tower is finite and, therefore, that $\Omega$ is finite, for if $\Omega$ were infinite a countably infinite tower of sets in $\Omega$ could be formed.

Suppose $h$ is in $L$. Then $\bigvee\{[n(h \vee \theta)] \wedge e\}, n=1,2,3, \cdots$, is in $L$ and is the characteristic function of the closure of $\{x \mid h(x)>0\}$.

Let $\Omega^{\prime}$ be the subcollection of $\Omega$ consisting of all the sets of $\Omega$ which contain no other set in $\Omega$. Then $\Omega^{\prime}$ is a partition of $S$. Suppose $k$ is in $L$ and $k$ has the values $y_{1}$ and $y_{2}\left(y_{1}<y_{2}\right)$ on some set (1) of $\Omega^{\prime}$. Let $h=$ $k-\left(y_{1}+y_{2}\right) / 2$ and $\gamma$ be the closure of $\{x \mid h(x)>0\}$. Thus $\gamma$ contains $k^{-1}\left(y_{2}\right)$ but not $k^{-1}\left(y_{1}\right)$ and as pointed out above $\gamma$ is in $\Omega^{\prime}$. But then $\gamma \cap \omega$ is in $\Omega^{\prime}$ and is a proper subset of $\omega$. This is a contradiction. Therefore $k$ is constant on $\omega$ and it follows that $k$ is a lineapcombination of the characteristic functions of the sets in $\Omega^{\prime}$. Thus $L$ is finite dimensional.

EXAMPLE 1. In the following all complements are taken with respect to $[0,1]$. Let $M_{1}$ be a Cantor set in $[0,1]$. For each positive integer $i>1$ take a Cantor set in each component of the complement of $M_{1} \cup \cdots \cup M_{i-1}$ and let $M_{i}$ be the union of these Cantor sets. Then for each positive integer $i$ let $H_{i}=M_{i} \cup[1 / i, 1]$. Suppose $x$ is a number in $[0,1]$ and $p$ is 
the smallest positive integer such that $x$ is in $H_{p}$. Let $f(x)=-p$ and $g(x)=-(p+1)$ if $p$ is odd and $g(x)=-p$ if $p$ is even.

Each of $f$ and $g$ is upper semicontinuous. The function $f-g$ takes on the values 0 and 1. If each of $f_{1}$ and $g_{1}$ is upper semicontinuous and $\left|f(x)-g(x)-\left(f_{1}(x)-g_{1}(x)\right)\right|<\frac{1}{4}$ for every number $x$ in $[0,1]$, then each of $f_{1}$ and $g_{1}$ is unbounded below.

An indication of the proof of the last statement is the following: Suppose $p$ is an odd positive integer, $k$ is a number such that $0<k<$ $1 /(p+2)$, and $w \in M_{p} \cap(0, k)$. Since $f_{1}(x)-g_{1}(x)>\frac{3}{4}$ for $x \in M_{p} \cap(0, k)$, $g_{1}(w)<f_{1}(w)-\frac{3}{4}$. As $g_{1}$ is upper semicontinuous there is an open interval $(a, b) \subset(0, k)$ containing $w$ such that every point of $g_{1}$ with abscissa in $(a, b)$ is beneath the horizontal line with ordinate $f_{1}(w)-\frac{3}{4}$. Because $w$ is a limit point of $M_{p+1},(a, b)$ contains a number $v$ of $M_{p+1}$. Thus $g_{1}(v)<$ $f_{1}(w)-\frac{3}{4}$. Therefore since $f_{1}(v)-g_{1}(v)<\frac{1}{4}, f_{1}(v)<f_{1}(w)-\frac{1}{2}$. As $f_{1}$ is upper semicontinuous, there is an open interval $(c, d) \subset(a, b)$ containing $v$ such that every point of $f_{1}$ with abscissa in $(c, d)$ is beneath the horizontal line with ordinate $f_{1}(w)-\frac{1}{2}$. Because $v$ is a limit point of $M_{p+2},(c, d)$ contains a number $u$ of $M_{p+2}$ and $f_{1}(u)<f_{1}(w)-\frac{1}{2}$.

The following example was suggested by the referee.

EXAMPLE 2. Let $X$ be an uncountable set and let $L$ be the space of all real valued functions on $X$ that are constant on the complement of some finite set depending on the function. Then $L$ is not $\sigma$-complete, it is infinite dimensional and the constant function 1 is a strong unit and has property c.

\section{BIBLIOGRAPHY}

1. S. Kempisty, Sur l'approximation de fonctions de première classe, Fund. Math. 2 (1921), 131-135.

2. S. Mazurkiewicz, Sur les fonctions de classe 1, Fund. Math. 2 (1921), 28-36.

3. W. Sierpiński, Demonstration d'un théorème sur les fonctions de première classe, Fund. Math. 2 (1921), 37-40.

4. A. C. Zaanen, Stability of order convergence and regularity in Riesz spaces, Studia Math. 31 (1968), 159-172. MR 39 \#1944.

Department of Mathematics, University of Houston, Houston, Texas 77004 